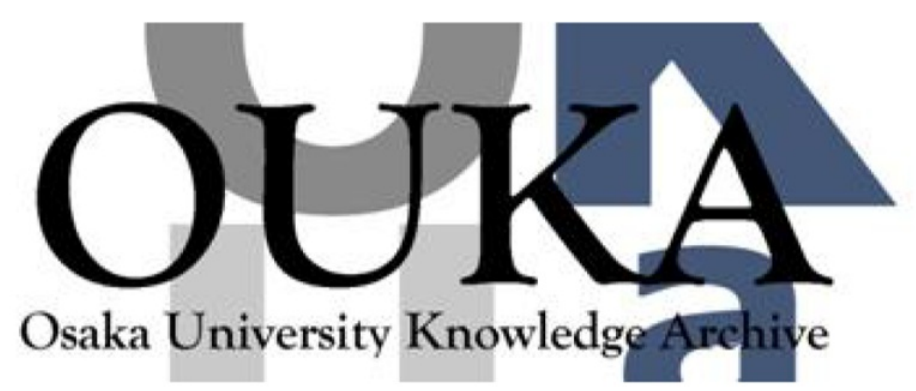

\begin{tabular}{|c|c|}
\hline Title & $\begin{array}{l}\text { ULtrasonic attenuation and grain-size } \\
\text { evaluation using electromagnetic acoustic } \\
\text { resonance }\end{array}$ \\
\hline Author (s) & Ogi, Hirotsugu; Hirao, Masahiko; Honda, Takashi \\
\hline Citation & $\begin{array}{l}\text { Journal of the Acoustical Society of America. } \\
98(1) \text { p. } 458-p .464\end{array}$ \\
\hline Issue Date & $1995-07$ \\
\hline oaire:version & VoR \\
\hline URL & https://hdl. handle. net/11094/84150 \\
\hline rights & $\begin{array}{l}\text { Copyright } 1995 \text { Acoustical Society of America. } \\
\text { This article may be downloaded for personal use } \\
\text { only. Any other use requires prior permission } \\
\text { of the author and the Acoustical Society of } \\
\text { America. }\end{array}$ \\
\hline Note & \\
\hline
\end{tabular}

Osaka University Knowledge Archive : OUKA

https://ir. Library. osaka-u. ac. jp/

0saka University 


\title{
Ultrasonic attenuation and grain-size evaluation using electromagnetic acoustic resonance
}

\author{
Hirotsugu Ogi, Masahiko Hirao, and Takashi Honda \\ Faculty of Engineering Science, Osaka University, Toyonaka Osaka 560, Japan
}

(Received 19 December 1994; accepted for publication 21 February 1995)

\begin{abstract}
Electromagnetic acoustic resonance is applied for determining the frequency dependence of the ultrasonic attenuation and the average grain size of low-carbon steels. Use of a noncontacting electromagnetic acoustic transducer (EMAT) makes it possible to isolate the attenuation within the plate specimens. The method relies on the Lorentz force mechanism to couple the EMAT to the specimen surfaces and then eliminates the other losses, which may otherwise occur with the contacting piezoelectric transducers. The measurement is independent of the EMAT used, the specimen thickness, the surface condition, the lift-off, etc., and is stable because of the noncontacting nature. First, the resonant frequencies are measured, to the accuracy of $10 \mathrm{~Hz}$, by sweeping the operating frequency and obtaining the amplitude spectrum over a band in the 0.5 20-MHz range. The ringing signals are excited and received by a shear wave EMAT and then processed with a superheterodyne receiver. Second, the attenuation coefficient as a function of the resonant frequency is determined. At each resonant frequency, the output signal rings down exponentially with time and the attenuation coefficient is obtained from the time constant by fitting an exponential decay to them. After correcting for the diffraction effect, the average grain size is obtained from the fourth-power term in the frequency dependence. The final results are favorably compared with the average of the three-dimensional grain-size distribution of steels. (C) 1995 Acoustical Society of America.
\end{abstract}

PACS numbers: $43.35 . \mathrm{Cg}$

\section{INTRODUCTION}

Grain size of a polycrystalline metal is one of the key factors that govern the mechanical properties like yield stress and fracture toughness. ${ }^{1}$ Many techniques have been studied for the nondestructive evaluation of grain size, including ultrasonic attenuation, x-ray diffraction, and magnetic tests. Among these, the ultrasonic technique is the most promising one, because it senses the through-thickness average of the grain structure on which the mechanical properties mainly * depend.

An ultrasonic wave propagating in metal is scattered by grains due to their different crystallographic orientations, causing amplitude loss in pulse-echo measurement. The relationship between the grain size and ultrasonic attenuation has been a principal topic ${ }^{2-7}$ in the area of ultrasonics. The classical study showed the fourth-power dependence of the attenuation on frequency in the region where the grain size is much smaller than the probe wavelength (the Rayleigh scattering). Although there has been a considerable research effort on grain-size evaluation using the formula in the Rayleigh scattering region, ${ }^{3-5}$ it is still under investigation. An apparent problem is the grain-size distribution in real metals, for which the power-law relationships were modified. ${ }^{6,7} \mathrm{We}$, however, consider that the main reason of the difficulty comes from the fact that the pure attenuation in the sample was not suitably extracted from as-measured quantity. Namely, in the attenuation measurement using a contacting transducer with well finished sample surfaces, the ultrasonic beam will lose its energy due not only to the attenuation in the sample (absorption and scattering) but also to the follow- ing four factors, (i) damping in the transducer, the couplant, and the buffer, if any, (ii) the reflection and transmission losses at the interfaces, (iii) the energy leakage into the transducer on reception, and (iv) the beam spreading (diffraction). Being interested only in the attenuation in the sample, we must remove factors (i) -(iv) from the as-measured attenuation by proper correction procedures. Diffraction effects can be eliminated by the familiar formula in pulse-echo measurements, ${ }^{8} 10$ while the correction for factors (i)-(iii) has not been successful so far in reality, because the acoustic parameters of all the components involved have to be determined a priori. It is not practical to know, for example, the thickness and the acoustic velocity of the couplant, which depend on the temperature, the applied pressure, the surface condition, etc.

In this paper, a new method of measuring the ultrasonic attenuation is proposed, which employs electromagnetic acoustic resonance (EMAR). ${ }^{11-14}$ and the technique is illuminated by the nondestructive evaluation of the grain size in steels. EMAR is a combination of the resonance method and the electromagnetic acoustic transducers (EMATs). At a resonance, many reflection echoes coherently overlap each other, which serves to provide an easily measurable signal intensity, compensating in excess for the inefficient transduction with EMATs. Use of a noncontacting EMAT for measurement has a pronounced advantage of eliminating the extra energy losses, which otherwise occur with the conventional contacting or immersion experiments based on the piezoelectric transducers. Measurement with an EMAT is inherently free from losses related to the interfaces because of the contactless coupling, so that the energy loss arises only from the 


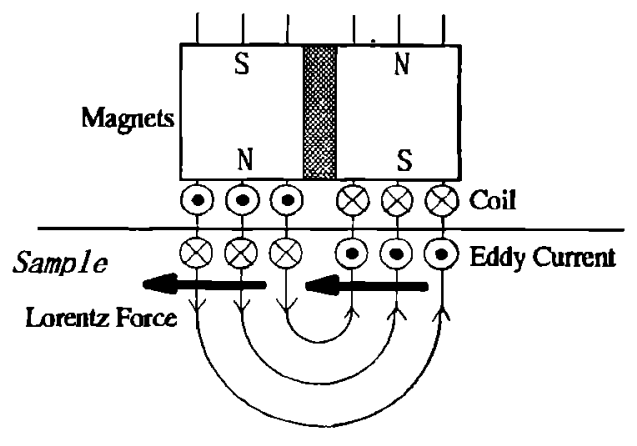

FIG. 1. Lorentz force mechanism of shear wave EMAT.

attenuation through the sample, the diffraction effect, and additionally the electromagnetic loss. The electromagnetic loss occurs when the elastic wave travels through the magnetic field and induces the eddy currents, which is, however, shown to be negligible compared with attenuation. Also, we develop a correcting algorithm for the diffraction loss at a resonance. It is then possible to evaluate an absolute value of ultrasonic attenuation based on EMAR. Furthermore, the larger number of echoes involved in the resonance, unlike a couple of echoes in the pulse-echo experiments, contributes to improve the accuracy and the reproducibility to a marked extent. A measurement may be completed within a very short time without any preparation on the sample surfaces.

For grain-size evaluation, we use the dependence of the attenuation on frequency $(f)$, following Mason and McSkimin, ${ }^{3}$ that is, the attenuation coefficient consists of the absorption term proportional to $f$, and the scattering term proportional to $f^{4}$. Attenuation measurement is performed on steel samples by changing the carbon content, pearlite fraction, martensite fraction, surface roughness, or texture. The final results are favorably compared with the average of the three-dimensional distribution of grain size calculated from the photomicrographs of the exposed cross sections of the samples.

\section{SHEAR WAVE EMATS}

The shear wave EMATs are used throughout this study (Fig. 1). The EMAT has a pair of permanent magnets, which have the opposite magnetization directions normal to the sample surfaces, and a flat elongated coil. When the coil is placed near the surface of a conducting material and is driven by an if burst current, eddy currents are induced in the near surface region of the sample. These currents interact with the static magnetic field applied by the magnets and generate the Lorentz forces upon electrons carrying the eddy currents. Through the collision with ions and other transformation mechanisms, the Lorentz forces are coupled to the mechanical body forces and generate an ultrasonic vibration. ${ }^{15}$ It is important that the wave source originates in the material, not in the transducer, eliminating the need for transmission. For the EMAT with the geometry in Fig. 1, the direction of the Lorentz force is principally parallel to the surface and results in the polarized shear wave propagating the thickness direction of the sample. The receiving principle is based on the reverse process of the generation.

\section{ISOLATION OF ULTRASONIC ATTENUATION}

When an EMAT is used, an observed attenuation coefficient $\alpha_{m}$ consists of the attenuation in sample $\alpha$, the diffraction loss $\alpha_{d}$, and the electromagnetic loss $\alpha_{e}$; that is, ${ }^{16}$

$$
\alpha_{m}=\alpha+\alpha_{d}+\alpha_{e}
$$

The electromagnetic loss occurs when the ultrasonic wave travels through the static magnetic field and the inverse Lorentz force mechanism gives rise to the eddy currents in the material. A part of these eddy currents is picked up by the coil in the EMAT, producing a received signal. The quantitative expression for $\alpha_{e}$ becomes $^{16}$

$$
\alpha_{e}=\frac{\sigma B_{a}^{2}}{2 \rho}
$$

where $\sigma$ is electrical conductivity of the sample material, $B_{a}$ the average static magnetic-flux density through thickness of the sample applied by the permanent magnets, and $\rho$ the mass density. Considering steel, for example, where $B_{a}=0.1$ $\mathrm{T}, \quad \sigma=3.0 \times 10^{-6} \mathrm{~S} / \mathrm{m}$, and $\rho=7900 \mathrm{~kg} / \mathrm{m}^{3}$, we have $\alpha_{e^{\prime}}=2 \times 10^{-6} \mu \mathrm{s}^{-1}$, while, as shown later and in a previous report, ${ }^{13} \alpha$ is larger than $10^{-3} \mu \mathrm{s}^{-1}$ in most steels for frequencies beyond $1 \mathrm{MHz}$. This leads to the estimation that $\alpha_{\boldsymbol{e}} / \alpha<0.2 \%$. We then ignore $\alpha_{e}$ henceforth in this paper. However, $\alpha_{e}$ must be taken into account for a material with a high conductivity, for a strong magnetic field, and/or for extremely low attenuating materials.

Isolation of $\alpha$ proceeds in three steps. First, we measure a resonant frequency. Second, we determine the relaxationtime coefficients at the measured resonant frequency. Finally, we correct for the diffraction effect by an iterative process.

\section{A. Measurement of relaxation-time coefficient}

Figure 2 shows the measurement setup, which is based on the computer-controlled spectrometer system produced by RITEC, Inc. Resonance frequencies are easily measured by activating the EMAT with long, high-power if bursts gated coherently, sweeping the operation frequency, and acquiring the amplitude spectrum. ${ }^{12-14}$ The input rf burst is much longer than the round-trip time in the sample plate. The amplitude spectrum is calculated from the in-phase and quadrature outputs of overlapped echoes (reverberation) after the analog superheterodyne processing. We then bring the sample plate into the ultrasonic resonance by driving the EMAT with the measured resonant frequency. At a resonance, all echoes reflected at the sample surfaces become coherent; that is, the echoes have a constant phase regardless of the echo number $i$, giving a productive interference among them. In this case, the in-phase integrator outputs $\left(\Sigma A_{i} \cos \Phi_{i}\right)$ decay exponentially with time, depending on the time coefficient $\alpha_{m}$ as shown in Fig. 3. The outputs of quadrature components $\left(\Sigma A_{i} \sin \Phi_{i}\right)$ also decay with $\alpha_{m}$, but with much lower amplitude.

We measure the ringdown curve by sweeping a short integrator gate (instead of a long one for obtaining the spectra) along the time axis, integrating both the in-phase and quadrature outputs, and calculating the root of the sum of the squares of these responses. ${ }^{13}$ Figure 4 presents an example of the measured ringdown curve for 6-mm-thick carbon steel at 


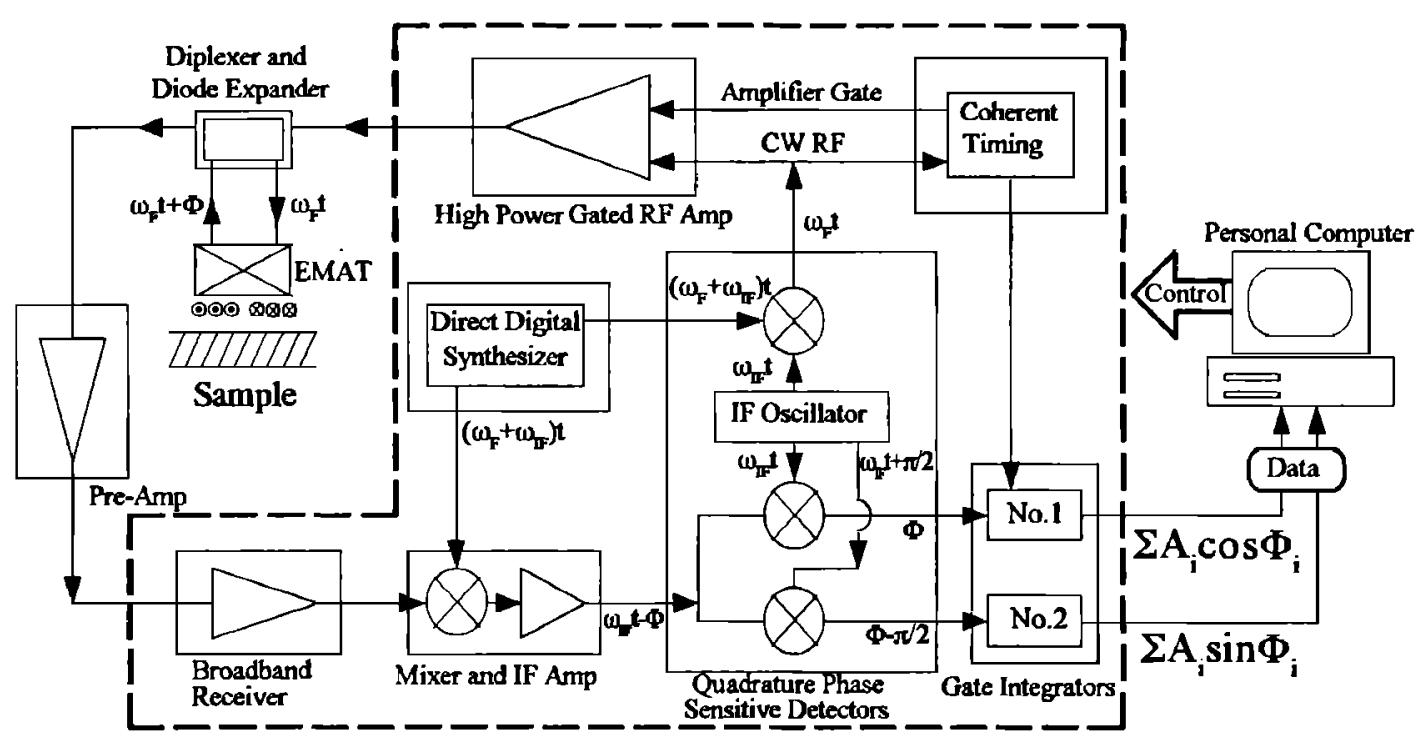

FIG. 2. Detection of amplitude spectrum and attenuation coefficient by superheterodyne receiver. The diagram includes phase modulation at each step. $A_{i}$ is the amplitude of the $i$ th reflected echo and $\Phi_{i}$ its phase.

the tenth resonant frequency around $2 \mathrm{MHz}$ [also in Fig. 8(b)]. We obtain the relaxation time coefficient $\alpha_{R}$ at the resonance, which is defined as the exponential decay constant of the ringdown curve, by fitting an exponential curve to it. We numerically simulated the reverberation signal using $\alpha_{R}$ as the damping coefficient $\left(\alpha_{m}\right)$ of individual reflection echoes in Fig. 3. The resultant ringdown signal reproduces $\alpha_{R}$ as the exponential decay constant, showing that $\alpha_{R}=\alpha_{m}$. This is true when the input burst is much longer than the round-trip time $T$ and the integrator gate is sufficiently short.

The resonant sharpness (or $Q$ value) also indicates $\alpha_{m}$. Its measurement can be done quickly only through the resonance spectrum. However, the $Q$ value is a derived quantity based on the geometry of the resonance measurement (EMAT, sample thickness, lift-off,...). Moreover, the diffraction effect on the $Q$ value is unclear.

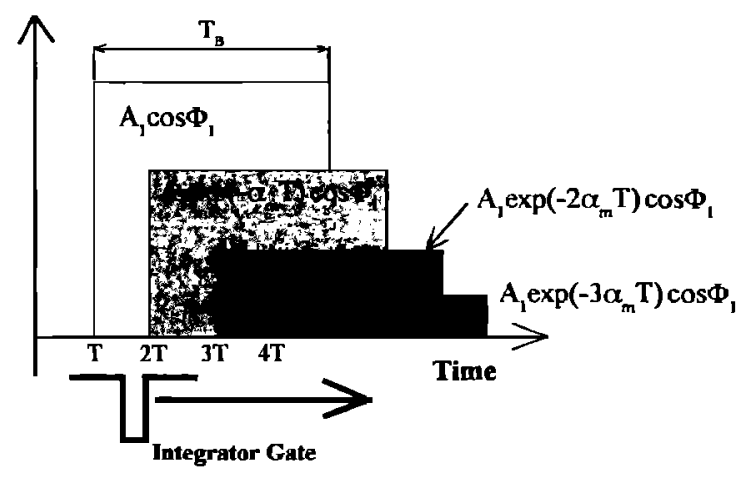

FIG. 3. Time response of coherent signals at a resonance after superhelerodyne processing (in-phase components). $A_{1}$ is the amplitude of the first echo and $\Phi_{1}$ is its phase shift. $T$ is the round-trip time in the sample and $T_{B}$ the width of the input of burst.

\section{B. Correction for diffraction loss}

Ultrasonic beam radiated from a finite transducer spreads perpendicular to the propagation direction and part of the incident energy will not return to the sending transducer. This diffraction causes the amplitude losses and phase shifts in the received echo signals. Following Seki et al., ${ }^{8}$ several authors ${ }^{9.10}$ have studied the phenomena for longitudinal waves radiated from a circular piston source transducer. But the existing solution is inadequate for the EMAR because of the noncircular geometry, a strength distribution over the radiating area, and highly overlapped echoes at a resonance.

\section{Diffraction phenomena radiated by an EMAT}

The Lorentz force induced by an EMAT has a threedimensional distribution in the sample in general. With the help of the exact distribution available from the nonlinear FEM computation, ${ }^{17}$ we here simplify the distribution to be two dimensional on the sample surface, which is characterized by a pair of parabolic curves with the nodes at the edges and the centerline (see Fig. 1). ${ }^{18}$ This simplification is allowed when the sample material has good electrical conduc-

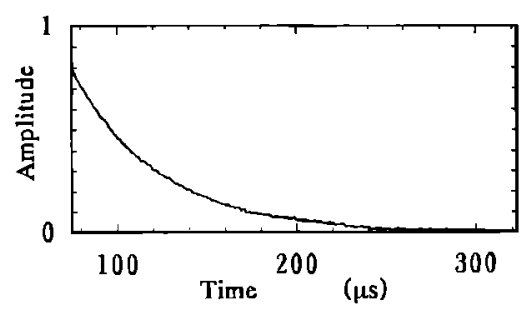

FIG. 4. Measured ringdown curve with a low-carbon steel sample $(6 \mathrm{~mm}$ thick) at the tenth resonant frequency (around $2 \mathrm{MHz}$ ). 


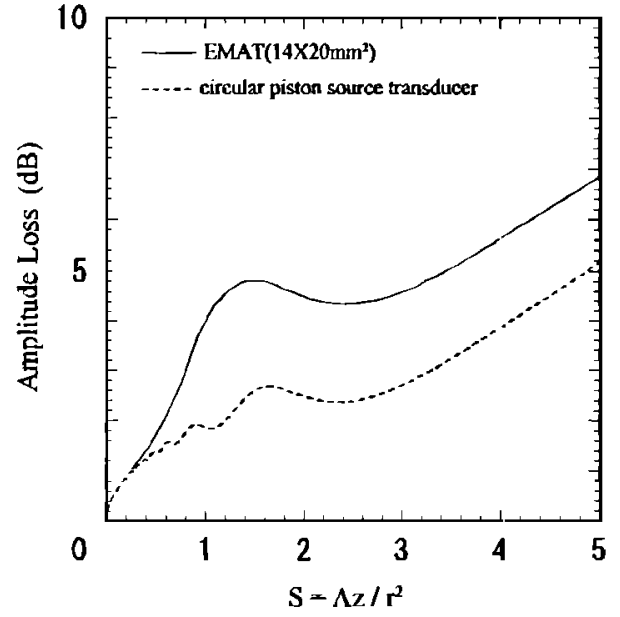

FIG. 5. Calculated amplitude loss due to diffraction for the shear wave EMAT with rectangular area of $14 \times 20 \mathrm{~mm}^{2}$ and the strength distribution of double-parabolic profile. Dotted line is the classical result for a circular piston source transducer by Seki ef al..$^{8} \Lambda$ denotes the wavelength, $z$ the propagation distance, and $r$ the equivalent radius of the radiating area.

tivity and high permeability because such in material has a very small electromagnetic skin depth. confi ing the Lorentz forces to the surface region. Assuming such a distribution of the shearing force on the radiating area, we simulate the three-dimensional ultrasonic diffraction to calculate the amplitude and phase profiles on the receiving area at a distance. The radiation into the sample is evaluated by integrating the radiation fields from all source elements, oscillating with the prescribed strengths, over the sending surface. ${ }^{18}$ The amplitude loss is reduced from the ratio of total power over the receiving area to that on the radiating one in a pulse-esho configuration. Figure 5 shows a calculated result for the shear wave EMAT, whose effective area is $14 \times 20 \mathrm{~mm}^{2}$. For a comparison, we also give the classical solution by Seki et al., ${ }^{8}$ which is no longer useful for correcting the diffraction loss for such a complicated transduction geometry as EMATs. The phase shift due to diffraction has little influence on the attenuation measurement, because the variation is $1 \mathrm{~m}-$ ited within the 0 to $\pi / 2$ range and it is asymptotic to the maximum $\pi / 2$ as $S\left(=\Lambda z / r^{2}\right)$ increases.

\section{Correction at a resonant state}

Figure 6 sketches the algorithm for correcting the diffraction effect at a resonance. Basically, the ringdown signal at a resonance is a superposition of reflected echoes of the resonant frequency with a constant phase. They are delayed by integer multiples of the round-trip time through the sample thickness $(T)$. Their amplitudes undergo damping due to the sample attenuation plus the diffraction effect (neglecting $\alpha_{e}$ ). The diffraction loss of each echo is provided by the diffraction data (Fig. 5), specific to the EMAT in use, using the resonant frequency and the propagation distance. The diffraction effect causes different losses to individual echoes, for they propagate different distances. Since all the echros are coherent, the shape of the ringdown curve is obtained by simply summing the echo amplitudes at a time delay.

The correction proceeds as follows:

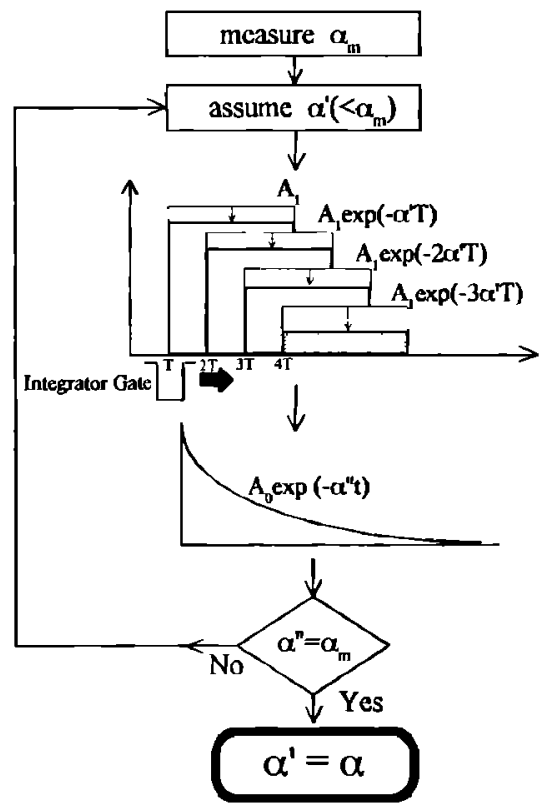

FIG. 6. Correction process for diffraction effect. The echo height is decreased in two steps, first following the diffraction-free rate $\left(\alpha^{\prime}\right)$ and then the diffraction loss of Fig. 5 .

(i) We measure a series of resonant frequencies and then the relaxation-time coefficient $\alpha_{m}$ at one of them.

(ii) We assume a trial time coefficient $\alpha^{\prime}\left(\alpha^{\prime}<\alpha_{m}\right)$, which should be free from the diffraction effect.

(iii) We calculate the amplitude of the $i$ th echo $E_{i}(t)$. which decreases at the rate of $\alpha^{\prime}$ as shown in Fig. 3: $E_{i}(t)$ can be expressed as

$$
\begin{aligned}
E_{i}(t)= & A_{1} H(t-i T) \exp \left\{-\alpha^{\prime}(i-1) T\right\} \\
& (i=1,2,3 \ldots),
\end{aligned}
$$

where $H(t)$ is the function defined by

$$
H(t)= \begin{cases}0, & t \leqslant 0 \text { and } t \geqslant T_{B}, \\ 1, & 0<t<T_{B},\end{cases}
$$

and $A_{1}, T$, and $T_{B}$ have been defined in Fig. 3 .

(iv) We give further damping to $E_{i}(t)$ by incorporating the calculated diffraction data in Fig. 5 .

(v) We then have the ringdown curve by numerically integrating these damped echoes overlapping heavily with a moving short gate. The gate width and the sweeping steps. as well as $T$ and $T_{B}$, follow the actual experiments. We obtain the time coefficient $\alpha^{\prime \prime}$ by again fitting the curve to an exponential decay. Now, if the trial $\alpha^{\prime}$ is the diffraction-free time coefficient, then $\alpha^{\prime \prime}$ must equal as-measured $\alpha_{m}$, because $\alpha^{\prime \prime}$ contains the diffraction effect. This series of calculations is repeated until $\left|\alpha_{m}-\alpha^{\prime \prime}\right| / \alpha_{m}<10^{-4}$ is reached.

\section{GRAIN-SIZE EVALUATION}

\section{A. Frequency dependence of attenuation}

Generally, the attenuation of an ultrasonic wave propagating in a sample originates from absorption and grain scattering. Absorption is related to dislocation mobility, ${ }^{19}$ thermoelastic effects, ${ }^{20}$ magnetic structure. ${ }^{21}$ interaction with electrons and phonons, ${ }^{22}$ and interaction with electron or 
nuclear spin. ${ }^{23}$ But at room temperature and a low frequency of the megahertz order, the effects of electrons and phonons and electron or nuclear spin are negligible. Furthermore, using only shear waves makes the thermoelastic effect almost inoperative. ${ }^{20}$ Consequently, the dislocation damping and the magnetoelastic effects only are responsible for the absorption. The relationship between the dislocation mobility and the frequency dependence of the attenuation shows the second-power dependence in low frequencies. ${ }^{19}$ But the very narrow range of frequencies used here $(1-10 \mathrm{MHz})$ allows the simplification of a linear dependence on frequency. The magnetoelastic effect also shows a linear function of frequency. ${ }^{20}$ We therefore consider that the attenuation due to the absorption effects is proportional to frequency. On the other hand, the attenuation caused by grain scattering in the Rayleigh region shows the fourth-power dependence on frequency. ${ }^{24}$

Finally, we employ the frequency dependence of the shear wave attenuation,

$$
\alpha(f)=a f+S D^{3} f^{4},
$$

where $a$ is the absorption factor, $S$ the scattering factor, and $D$ the average grain size. We can separate the absorption and the scattering parts in $\alpha(f)$ from their different dependence on $f$. Bhatia ${ }^{24}$ derived scattering factors for longitudinal and shear waves. His theoretical result shows that grain scattering occurs more intensively for the shear wave than for the longitudinal wave, making it advantageous to use the shear wave for grain-size evaluation. On the other hand, the attenuation by absorption is smaller for shear waves than for longitudinal waves because of the thermoelastic effect acting only for longitudinal waves. Therefore the separation of absorption and scattering effects is much easier for the shear wave, resulting in more accurate evaluation of grain size. From the experimental side, the shear wave resonance is most easily detected with an EMAT of this simple structure (Fig. 1).

\section{B. Samples}

To demonstrate the usefulness of the EMAR method, we measured the grain size of various low-carbon steel samples to see the influences of:

carbon content: 13 samples with three different carbon contents of 0.007 mass $\%, 0.062$ mass $\%$ and 0.147 mass\%;

(ii) pearlite fraction: four samples with $2 \%, 61 \%, 80 \%$, and $98 \%$ pearlite volume fractions;

(iii) martensite fraction: 12 samples with four different martensite volume fractions of $15.3 \%, 20.5 \%, 30.9 \%$, and $39.4 \%$;

(iv) surface roughness: seven samples with different average roughness ranging from $R_{a}=0.14$ to $8.7 \mu \mathrm{m}$; and

(v) texture: three samples with the shear wave velocity difference of $1.58 \%, 3.00 \%$, and $5.19 \%$.

All samples were sized $6 \times 100 \times 100^{w} \mathrm{~mm}^{3}$. Naturally, each sample possesses a grain-size distribution. The photomicrographic examination only reveals the two-dimensional (2-D) distribution on an exposed cross section. The average

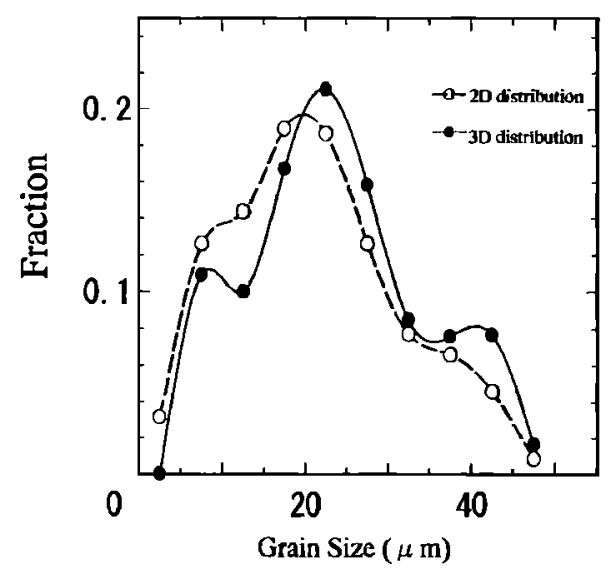

FIG. 7. Calculation of three-dimensional grain-size distribution.

size determined from such an examination is smaller than that of the three-dimensional (3-D) distribution, which substantially influences the mechanical characters of the bulk material. The grain diameter appearing on the observation plane is smaller than the real diameter of the grain. Especially, for a grain structure which is far from being equiaxial, the cross-sectional diameter is more likely to underestimate the average grain size. We calculated the 3-D distribution from the measured 2-D distribution by applying the model in which the grain shape is a regular polyhedron or semiregular one whose number of faces is determined only from the size, and each grain has the unique diameter distribution on the exposed cross section depending on the number of the faces. ${ }^{25}$ An example of the calculated 3-D distribution is shown in Fig. 7. The average grain size in the 3-D distribution $(23 \mu \mathrm{m})$ became larger about $10 \%$ of that of the 2-D distribution $(21 \mu \mathrm{m})$. The fraction of the small grains in the optical observation also decreases in the calculated 3-D distribution, resulting in a narrower spread of diameter.

\section{Experiments}

In the measurement of a resonant frequency, the burst signal of $40-\mu$ s duration is used to excite the EMAT. The frequency is swept by $500-\mathrm{Hz}$ steps. The ringing signals were integrated with the gate of $200-300 \mu$ s long, involving approximately from 50 to 80 reflection echoes $(T \sim 3.7 \mu \mathrm{s})$. In the attenuation measurement, the integrator gate of $5 \mu \mathrm{s}$ long is swept at every $1 \mu \mathrm{s}$ from just after the excitation until the ringing completely fades. Figure 8 shows a measured series of resonant frequencies and the attenuation curves at three resonant frequencies for a steel sample. The frequency dependence of the attenuation is obtained from the relationship between the attenuation $(\alpha)$ and the resonant frequency, as shown in Fig. 9. We plotted both the as-measured coefficient $\alpha_{m}$ and the pure attenuation coefficient $\alpha$ after correcting for the diffraction effect. The corrected data $(\alpha)$ shows a better fitting to Eq. (5), especially in the low-frequency region. The frequency dependence of $\alpha$ approaches a linear dependence as the grain size decreases, showing the dominant absorption term over the scattering term in Eq. (5). We evaluated the average grain size by fitting Eq. (5) to the 

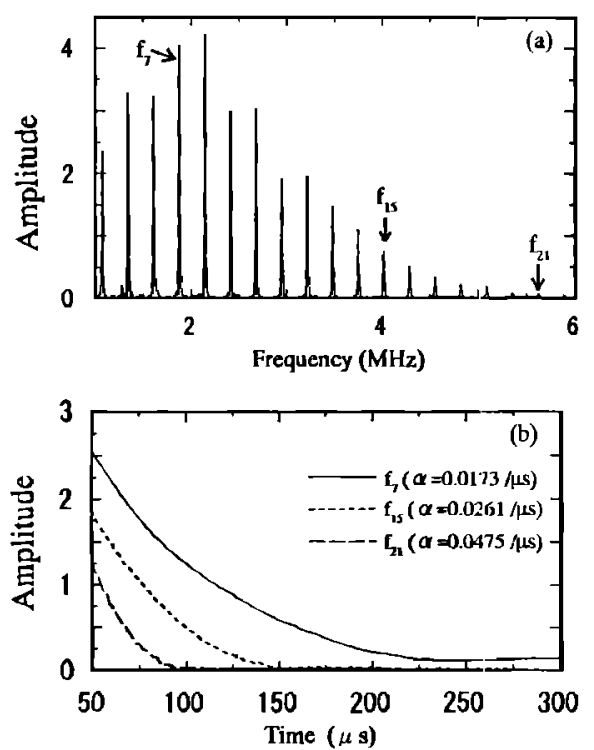

FIG. 8. Measurement of (a) resonant frequencies and (b) attenuation curves at three resonant frequencies.

measured $\alpha(f)$ and then extracting the term in $f^{4}$. With a suitable scattering factor $S$, the coefficient of the $f^{4}$ lerm provides the average grain size. Although the scattering factor can be derived theoretically, ${ }^{26}$ we calibrated $S$ to be $S=2.25 \times 10^{-10} \mu \mathrm{s}^{3} / \mu^{3}$ using as the reference a sitee] sample which has the narrowest distribution of grain size.

Papadakis $^{27}$ pointed out that $D^{3}$ in Eq. (5) should be replaced by $\left(D^{6}\right) /\left(D^{3}\right) .\langle\rangle$ being the average. We applied this formula to the present experiments with the inferred 3-D distribution grain size and the recalibrated $S$. The EMAR result, however, gave a much smaller grain size than that determined from the photomicrographic method. We find that the use of $\langle D\rangle^{3}$ for $D^{3}$ in Eq. (5) leads to the most acceptable agreement with the photomicrographic examination. The grain sizes evaluated thus ( $\left.D_{\mathrm{EMAR}}\right)$ are compared with those of the optical measurement after converting to the 3-D distribution $\left(D_{\mathrm{PHOT}}\right)$ in Fig. 10.

\section{Discussions}

The grain sizes evaluated by EMAR well agree with those by photomicrographic method within an error band of

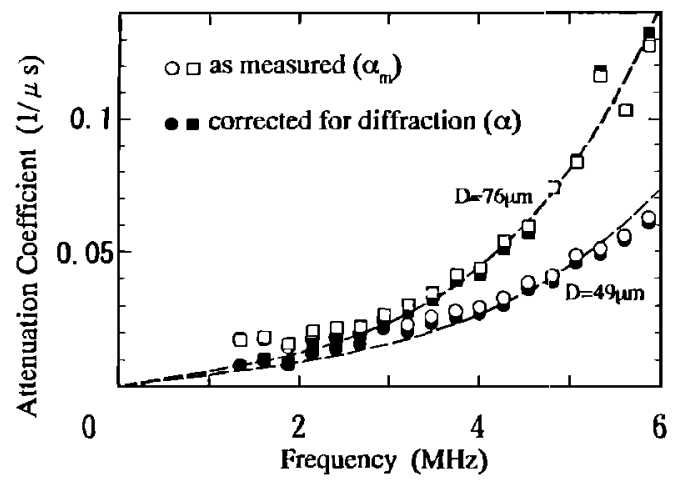

FIG. 9. Measurement of trequency dependence of shear wave attenuation.

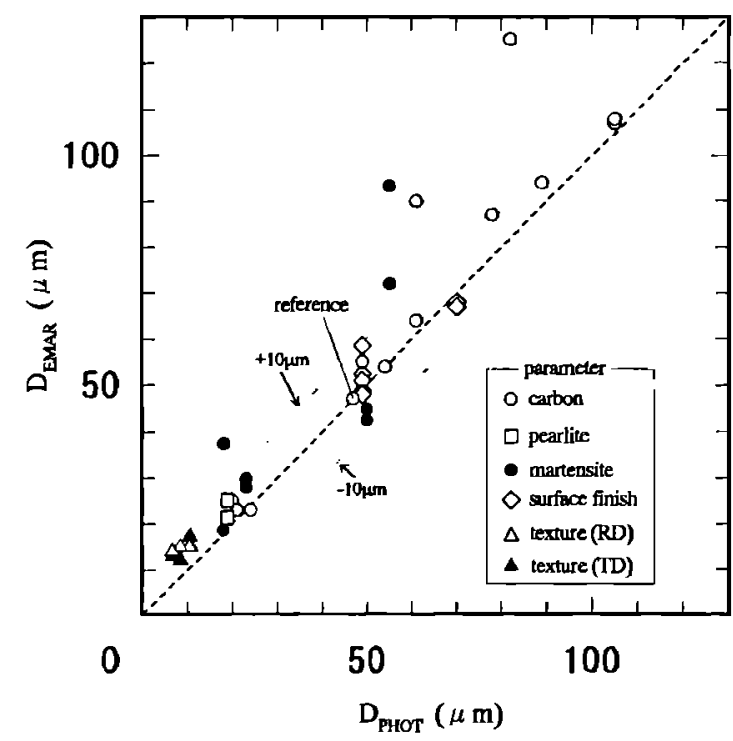

FIG. 10. Results of measured grain sizes. The horizontal axis denotes the 3-D average grain sizes determined from photomicrographs and the vertical axes are those measured with the EMAR method.

$6 \mu \mathrm{m}$. But there are some outlying points. The unfavorable results with the samples containing the martensitic phase is explained from two points. First. the definition of "grain" is substantially different between the photomicrographic method and the EMAR. The former recognizes grain simply from the boundaries of components, but the latter defines it from the elastic discontinuity. The sizes estimated by the two methods therefore do not necessarily agree with each other, especially when there is a hierarchy in the metallurgical structure like martensitic steel. Second, the dual phase (martensite-ferrite) system introduces another scattering problem. In the sample with two phases, the scattering occurs not only due to elastic anisotropies within a phase but due to the phase boundaries. ${ }^{28}$ This means that Eq. (5), derived from the single phase theory, is no longer usable. The favorable results for the samples containing pearlite, which are also of the dual phase system (pearlite and ferrite), suggest the nearly equal elastic properties between the pearlite and ferrite structures.

There are two more samples that show greater $D_{\text {EMAR }}$ than $D_{\text {PHOT }}$ among those of changing carbon content. These samples both possess broad distributions of grain size, involving plural peaks. Since Eq. (5) strictly applies to the hypothetical polycrystalline material composed of single size grains, the formula becomes progressively inadequate as the distribution broadens.

Surface roughness can cause the extra attenuation, following the $f^{2}$ law. ${ }^{29}$ Generally, the separation between the attenuation due to the surface roughness and that by grain scattering is difficult because of the fairly close dependence on frequency $\left(f^{2}\right.$ and $\left.f^{4}\right)$. The effect is more likely to be included in the scattering term in Eq. (5) overestimating the grain size. But, in Fig. $10, D_{\text {EMAR }}$ is larger than $D_{\text {PHOT }}$ by only $8 \mu \mathrm{m}$ even for the roughest surfaces of $R a=8.7 \mu \mathrm{m}$. The effect of surface roughness is then insignificant for the present set of samples.

For the samples with texture, two shear waves polarized 
in the orthogonal directions are independently used for the measurement, but no significant effects of texture on the grain-size evaluation are observed.

\section{CONCLUSION}

EMAR is revealed to be ideally suited to the nondestructive evaluation of ultrasonic attenuation. Owing to the noncontacting EMATs, it conveniently excludes the interfering effects which occur with the conventional techniques. The diffraction effect can be strictly corrected using the numerical iteration procedure, resulting in an absolute evaluation of the attenuation coefficient. The measurement can be done with great ease and high reproducibility, accommodating rusty or moderately rough surfaces as well.

The EMAR technique was applied for the grain-size determination of steel samples. Grain size evaluated by EMAR showed good agreement with those estimated by photomicrographic measurements within an error budget of $6 \mu \mathrm{m}$, with some exceptions. The measurement did not suffer from carbon content, pearlite content, surface roughness (up to $R a=8.7 \mu \mathrm{m}$ ), and texture. The unfavorable evaluations took place for samples containing the martensite phase, which is caused by unclear observation of grain boundaries in the photomicrographs. The technique gives too large average grain size for samples with broad distributions of grain size.

EMAR, however, is restricted to the plate geometry of samples to support multiple reflections. The diffraction correction is unavailable for other geometries at present. In case of a thick sample, the intervals of neighboring resonant peaks become narrower and eventually they overlap each other, making the resonant frequency measurement inaccurate or infeasible. When the plate is too thin, the resonant peaks are dispersed. The frequency dependence of $\alpha$ is measured at a few resonant frequencies, and the fit to Eq. (5) will not be successful. The measurable thickness is between 0.5 and $50 \mathrm{~mm}$ for common metals, but it depends mainly on the attenuation character itself. Discrete measurement of attenuation at the resonances might be a problem. Interpolation is available to obtain attenuation for intermediate frequencies.

\section{ACKNOWLEDGMENTS}

We are grateful to Dr. Gary L. Petersen, RITEC Inc., for the kind assistance and the valuable discussions on the resonance measurement with EMATs. The sample steels were supplied courtesy of the Committee of Sensors for Microstructures, Iron and Steel Institute of Japan.

'E. O. Hall. Yield Point Phenomena in Metals and Alloys (Plenum, New York, 1970).

${ }^{2}$ L. Rayleigh, The Theory of Sound I (Dover, New York, 1945), p. 149.

${ }^{3}$ W. P. Mason and H. J. McSkimin, J. Acoust. Soc. Am. 19, 464-473 (1947).

${ }^{4}$ E. P. Papadakis, J. Acoust. Soc. Am. 37, 711-717 (1965).

${ }^{5}$ R. L. Smith, W. N. Reynolds, and H. N. G. Wadley, Met. Sci. 15, 554-558 (1981).

${ }^{6}$ E. P. Papadakis, J. Appl. Phys. 35, 1586-1594 (1964)

${ }^{7}$ D. Nicoletti, N. Rilgutay, and B. Onaral, J. Acoust. Soc. Am. 91, 32783284 (1992).

${ }^{8}$ H. Seki, A. Granato, and R. Truell, J. Acoust. Soc. Am. 28, 230-238 (1955).

${ }^{9}$ H. J. McSkimin, J. Acoust. Soc. Am. 32, 1401-1404 (1960).

${ }^{10}$ E. P. Papadakis, J. Acoust. Soc. Am. 40, 863-876 (1966).

${ }^{11}$ H. Fukuoka, M. Hirao, T. Yamasaki, H. Ogi, G. L. Petersen, and C. M. Fortunko, Review of Progress in QNDE, edited by D. O. Thompson and D. E. Chimenti (Plenum, New York, 1993), Vol. 12, p. 2129-2135.

${ }^{12}$ M. Hirao, H. Ogi, and H. Fukuoka, Rev. Sci. Instrum. 64, 3198-3205 (1993).

${ }^{13}$ M. Hirao and H. Ogi, Appl. Phys. Lett. 64, 2217-2219 (1994).

${ }^{14}$ G. L. Petersen, C. M. Fortunko, B. B. Chick, and M. Hirao, Rev. Sci. Instrum. 65, 192-198 (1994).

15 J. J. Quinn, Phys. Lett. A 25, 522-523 (1967).

${ }^{16} \mathrm{H}$. Ogi, M. Hirao, T. Honda, and H. Fukuoka, Review of Progress in QNDE, edited by D. O. Thompson and D. E. Chimenti (Plenum, New York, 1995), Vol. 14.

${ }^{17}$ H. Ogi, M. Hirao, K. Minoura, and H. Fukuoka (to be submitted).

${ }^{18} \mathrm{H}$. Ogi, M. Hirao, T. Honda, and H. Fukuoka, to be published in J. Acoust. Soc. Am., in press.

${ }^{19}$ A. Granato and K. Lücke, J. Appl. Phys. 27, 583-593 (1956).

${ }^{20}$ K. Liicke, J. Appl. Phys. 27, 1433-1438 (1956).

${ }^{21}$ S. Levy and R. Truell, Rev. Mod. Phys. 25, 140-145 (1953).

${ }^{22}$ L. Mackinnon, Phys. Rev. 98, 1210-1210 (1955).

${ }^{23}$ J. J. Quinn and S. C. Ying, Phys. Lett. 23, 61-62 (1966).

${ }^{24}$ A. B. Bhatia, J. Acoust. Soc. Am. 31, 16-23 (1959).

${ }^{25}$ K. Matsuura and Y. Itoh, Mater. Trans. JMM 32, 1042-1047 (1991).

${ }^{26}$ A. B. Bhatia and R. A. Moore, J. Acoust. Soc. Am. 31, 1140-1141 (1959).

${ }^{27}$ E. P. Papadakis, J. Acoust. Soc. Am. 33, 1616-1621 (1961).

${ }^{28}$ C. F. Ying and R. Truell, J Appl. Phys. 27, 1086-1097 (1956).

${ }^{29}$ P. B. Nagy and J. H. Rose, J. Appl. Phys. 73, 566-580 (1993). 